\title{
Quantitative Microanalysis of Chromites and Garnets at Low kV Using Fe and Cr L $\alpha$ and L $\beta$ X-ray Lines
}

\author{
Anette von der Handt ${ }^{1}$, Aurélien Moy ${ }^{2}$ and John Fournelle ${ }^{1}$ \\ ${ }^{1}$ University of Minnesota, Minneapolis, Minnesota, United States, ${ }^{2}$ Department of Geoscience, University \\ of Wisconsin-Madison, Madison, Wisconsin, United States
}

Electron probe microanalysis (EPMA) is one of the most versatile instruments for Earth and materials research as it combines high analytical precision with high spatial resolution. The introduction of Schottky field emission emitters to EPMA instruments, generating bright and narrow electron beams, provides new incentive to push the current limits of quantitative microanalysis further into the sub-micron realm.

In particular, the field of experimental petrology sometimes struggles with growing large and homogeneous minerals. Furthermore, improved spatial resolution is absolutely required to analyze some experimental phases, such as those grown in the diamond anvil cell. Here, spinel grains are generally smaller than 3 microns and often even smaller than 1 micron, making reliable analysis difficult to impossible owing to secondary fluorescence [1]. Garnets suffer from similar problems in experimental petrology but in addition are also a mineral phase of wide interest for thermobarometric investigations in metamorphic rocks, often occurring as small mineral inclusions or as strongly zoned crystals.

Unfortunately, beam size alone does not control the extent of electron scattering within the sample, because electron beam potential and phase chemistry are also important. Hence, the beam accelerating voltage needs to be reduced from the traditional $15-20 \mathrm{kV}$ to 5-7 kV to achieve high spatial resolution for EPMA analysis. At these low $\mathrm{kV}$ conditions, $\mathrm{K} \alpha \mathrm{X}$-ray lines of the transition elements such as $\mathrm{Fe}$ and $\mathrm{Cr}$ are no longer generated, so L-family X-ray lines have to be utilized. However, the properties of these Xray lines are complicated by bonding effects that differ from one material to another, with behavior that is not fully understood. Applying the traditional method of quantification by using background-corrected L $\alpha$ peak intensities often results in inaccurate element concentrations.

Moy et al. [2, 3] showed that while using the Fe L $\alpha$ peak intensities and traditional quantification methods does not yield accurate element concentrations, using the integrated intensities of the combined Fe L $\alpha$ and L $\beta$ peak in combination with a calibration curve approach results in good quantitative data. They successfully applied this method to a wide range of iron silicides, sulfides and olivines using just Fe metal as calibration standard.

In this study, we investigated garnets and chromites covering a wide compositional spectrum at $7 \mathrm{kV}$ through traditional WDS using a TAPL monochromator crystal as well as the extended range Soft X-ray Emission Spectrometer (SXES, JEOL Inc.) on a JEOL JXA-8530FPlus electron microprobe. The SXES detector consists of two variable spacing diffraction gratings (JS300N, JS2000) and a Peltier-cooled CCD camera, with the advantage of acquiring simultaneously the entire X-ray spectrum within the energy range of the individual grating. The JEOL JS2000 extended range grating can detect X-rays in the range from $240 \mathrm{eV}$ to $2800 \mathrm{eV}$ and therefore covers the first order L-lines of $\mathrm{Cr}$ and Fe, enabling acquisition of data much faster than traditional WDS with its stepwise acquisition.

Fe and Cr L spectra were acquired with both detector types and the intensities of their respective L $\alpha$ and L $\beta$ peaks integrated after background correction. Area k-ratios were calculated with reference standards 
of known composition and the resulting calibration curves were used for quantification. Area k-ratios of garnets vary smoothly as a function of Fe content despite their wide compositional range. This allows each set of area k-ratios to be fitted by a second-order polynomial with a good coefficient of determination (Figure 1), similar to the previously studied mineral families [2,3]. In contrast to the previous studies, better results were obtained with an almandine (in this study Green's Creek Almandine, Harvard Collection \# 112140) as reference material instead of Fe metal or iron oxides. Area k-ratios for Cr L-lines in chromites were equally well fitted to a second-order polynomial (Figure 2a). Again, better results were obtained using a chromite standard (NMNH 117075 chromite) rather than Cr-metal. Most notably, quantification of chromite Fe-contents using a single reference material yielded two distinct calibration curves that depend on the overall chromite chemistry, best expressed by the $\mathrm{Cr}$-number $(\mathrm{Cr} / \mathrm{Cr}+\mathrm{Al})$ (Figure $2 \mathrm{~b})$. Work to date suggests that a spinel Cr-number of 0.5 is a suitable point of transition from one calibration curve to another [4].

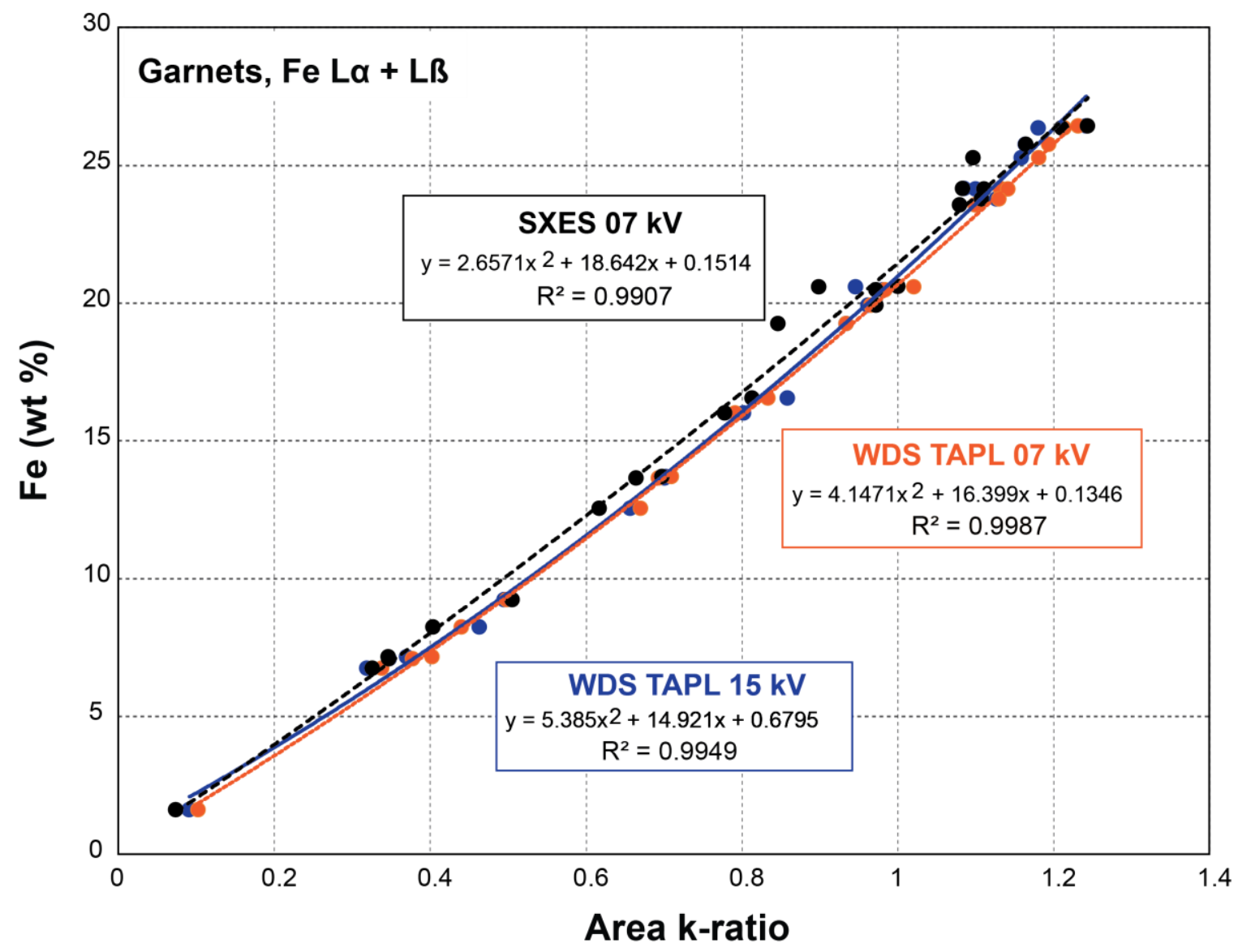

Figure 1. Figure 1. Area k-ratios of the Fe $\mathrm{L} \alpha$ - $\mathrm{L} \beta \mathrm{X}$-ray measured on garnets at $7 \mathrm{kV}$ and $15 \mathrm{kV}$ using a TAPL monochromator crystal and the SXES JS2000 grating. 


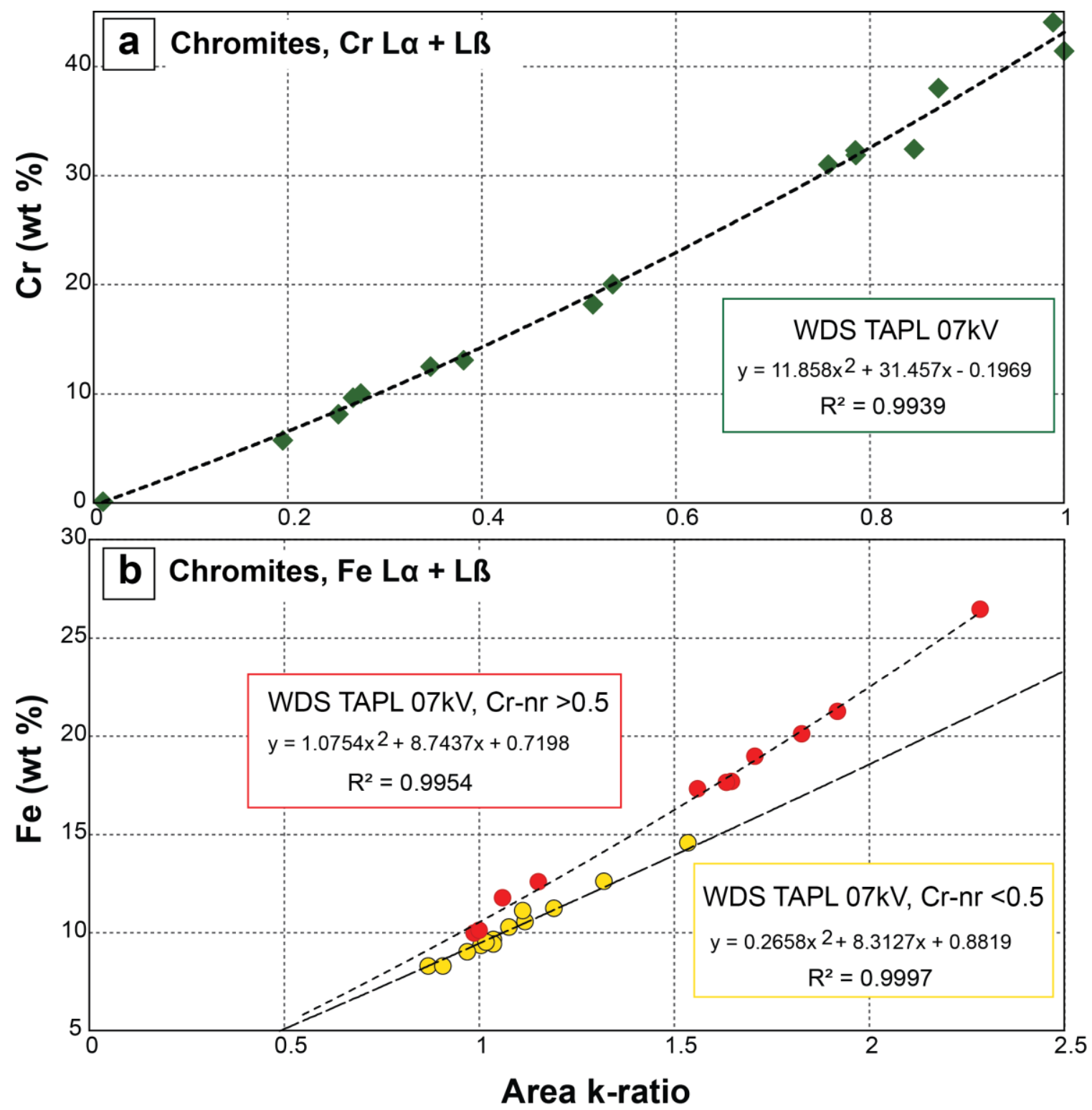

Figure 2. Figure 2. Area k-ratios of (a) $\mathrm{Cr}$ and (b) $\mathrm{Fe} \mathrm{L} \alpha-\mathrm{L} \beta \mathrm{X}$-ray lines measured at $07 \mathrm{kV}$ in chromites using a TAPL monochromator. A single second-order polynomial provides a good fit for the Cr L-lines for all chromite compositions while $\mathrm{Fe} \mathrm{L} \alpha-\mathrm{L} \beta$ area $\mathrm{k}$-ratios require two different calibration curves, one for chromites with $\mathrm{Cr}$-numbers $(\mathrm{Cr} /(\mathrm{Cr}+\mathrm{Al})>0.5$ and one with $\mathrm{Cr}$-numbers $<0.5$.

References

[1] FA Davis, E Cottrell (2018). American Mineralogist. 103 (7): 1056-1067.

[2] A Moy et al., (2019). Microsc. Microanal. 25 (3): 664-674.

[3] A Moy et al. (2019). American Mineralogist. 104 (8): 1131-1142.

[4] Support for this research came from the National Science Foundation: EAR-1849465 (AVDH), EAR1337156 (JHF), EAR-1554269 (JHF) and EAR-1849386 (JHF). 